\title{
Study on Open Source Software Based on Cloud Computing Platform
}

\author{
Long Zhu \\ Sichuan Information Technology College, Guangyuan, Sichuan, 628040, China
}

Keywords: Cloud computing platform, Open source software, Study

\begin{abstract}
With the constant development of science and technology and social progress, open source software provides the establishment of cloud computing platform for a lot of convenience and provides certain challenges for scientific and reasonable selection of application software. To analyze cloud computing platform effectively, it is necessary to fully understand and study open source software of cloud computing platform, analyze the practical situation of open source software of cloud computing platform, provide a foundation for further analysis on multi-angle open source software of service model and provide strong guarantee for the construction of cloud computing platform.
\end{abstract}

\section{Introduction}

In the current stage, the development of open source software of information cloud computing is a main future direction. The constant development of open source software promotes the progress of cloud computing and the development of cloud computing promotes the progress of open source software. They supplement each other. Many enterprises start to be committed to studying open source software of cloud computing. The fundamental objective of cloud computing application is to allocate resources freely, flexibly and reasonably. The development of open source software provides it with certain technical support. In particular, open source software is indispensable in some open cloud computing. That is to say, the soul of cloud computing is open source software which drives the development of cloud computing and gradually becomes an important objective and direction in future development.

\section{Basic introductions to cloud computing and open source software}

Open source software is source code software that can be obtained from computer. Common users can download software freely and commercial users can reduce the time and period of software development. Therefore, both business and individuals have started to use open source software widely. Cloud computing is a technology integrating internet technology, distributed calculation and large-scale management resources. It belongs to systematic research and application engineering, including computer safety, processing of mass data, virtual resources and management data center. Cloud computing can be divided into three parts, i.e. basic service layer (IaaS), software service layer (SaaS) and platform service layer (PaaS).Cloud computing can provide people with low-cost and convenient services. The development of open source software based on cloud computing will promote the development of cloud computing. Open source software can meet basic requirements of cloud computing. It has certain safety and transparency and is more convenient for the formation of cloud computing standard. Open source software can guarantee cloud computing technology with better expandability and flexibility ${ }^{[1]}$.

\section{Development status of open source software of cloud computing}

In the current stage, there is a lot of open source software of cloud computing, mainly including OpenStack, OpenNebula, CloudStack and Eucalyptus etc.

\section{Eucalyptus}

Eucalyptus open source software studied and developed by the Faculty of Computer Science, UCSB is a project applied early, which has many market shares in the current stage. Users may not 
modify components during use and it can be applied on unmodified GNU Linux core. Eucalyptus can provide compatible S3 and EC2 interfaces for cloud computing platform so that users can turn to public platform from private platform.

\section{OpenStack}

Cloud computing platform of OpenStack open source software studied jointly by USA Rsckspace and NASA mainly combines Cloud Files Platform of Rsckspace and Nebula Platform technology of NASA organically. With the support of many open source organizations and business companies, OpenStack is the technical support for future development of cloud computing open source software. With constant development, it has gradually become one of inter-integrated technologies of cloud computing.

\section{CloudStack}

VMOps Company studied CloudStack open source project in 2008 and changed CloudStack into Cloud.com in 2010. Their main difference is commercial version and community version. Citrix Company purchased Cloud.com in July 2011, integrated all branches of business maintenance appropriately in open source brand and established open source software project above and complete open source software project in Apache Software Foundation. It is a technology with large-scale application in the world in the current stage, such as GoDaddy and Aynga.

\section{OpenNebula}

RubenSMontero and Ignacio M Llorente established OpenNebula open source software project in 2005 and published public version in 2008. OpenNebula is actually a measure for solving open source project studied by basic equipment management of cloud computing and virtual enterprise data center as well as project framework with modularization, openness and expansibility. Currently, it has gradually developed into a core project of virtual execution environment management of cloud computing of European Union.

\section{Structure of open source software of cloud computing platform}

\section{Architecture of software in basic service layer (IaaS)}

Software in basic service layer is mainly cloud computing service system in the lowest layer, which mainly provides users with some infrastructures such as virtual network, virtual storage and VM. Currently, basic service layer can be roughly divided into two architectures: first, three-tier structure formed based on open source software represented by XED Clound and Eucalyptus. Second, two-tier structure formed based on open source software represented by ECP, Nimbus and Nebula ${ }^{[2]}$.

Two-tier architecture mainly includes work node layer and control layer. Work node layer forms through a series of work nodes and storage system and cloud controller form control layer. Cloud controller that can dispatch and manage work nodes on the whole platform is mainly used to connect cloud computing platform side and client, mainly including user manager, dispatcher, monitor, storage manager, cloud interface, network manager and platform component manager. The fundamental function of storage system is that it can store all impression files on the platform. For the access to cloud computing platform from client, browser and client interface are mainly used. Cloud interface can convert client command and form a uniform identification platform. Management components on the whole platform constitute platform component manager. Monitor is mainly used to monitor the practical situation of resources used in work nodes and provide a foundation and guarantee for subsequent load balancing and work node adjustment. User manager manages and identifies user identity. Storage manager connected to storage system can map the whole management platform, virtual disk file and snapshot file etc. Network manager manages virtual network on the whole cloud computing platform, including VPN and VLAN. Virtual machine manager operates in work node. Users can deploy VM cases reasonably on VMM and form VM application software environment. VM cases on the platform can be managed with VMM, such as migration VM and registration VM.

In terms of system, the main difference of three-tier structure is that a middle layer of cluster control node is added. Basic functions of the middle layer include the following: first, cluster control system of network port management is mainly established in cluster DNS and DHCP servers. Second, 
it monitors resource use situation of cluster node and reports node monitoring information to superior controller. When cloud controller invokes work nodes at the bottom, it is necessary to refer to the monitoring information of cluster control nodes. Third, it has router function and can interconnect two cluster work nodes and communicate with them. In terms of function, three-tier architecture has better expansibility than two-tier architecture. In two-tier structure, work nodes are directly managed by cloud controller and VM can be deployed faster. In three-tier structure, work nodes and cluster controller can be communicated directly. Work nodes can be communicated based on cloud controller and cluster controller. Cloud controller in middle layer can adjust work nodes, reduce expenditures of cloud controller to a certain extent and improve the expansibility of the platform.

\section{Architecture of software in software service layer (SaaS)}

Software in software service layer mainly provides services for users and makes deployment in platform service layer and basic service layer. Main representatives of software service layer architecture are OpenId and Zimbra, mainly including storage system, application node system and cloud controller system. Cloud controller mainly includes the following components: monitor, pplication manager, metadata manager, user manager, platform component manager, database manager and cloud interface. Metadatabase manager is used to manage platform data. Users can access the whole platform with browser. Application manager can manage the practical situation of operating software, e.g. balanced platform load and accessed platform dispatching process. Database manager is mainly used to control storage system so as to achieve the purpose of managing application data platform. Software service layer platform has user manager, monitor and platform component manager functions similar to platform service layer and basic service layer. Cloud controller has the function of application distribution in node. Generally, a software service layer platform does not simply provide an application for users. For example, Zimbra not only has chat function, but also can manage users' email. Functions above might exist on the same physical device or multiple devices. User platform does not attach importance to running program. It only provides certain application for the platform ${ }^{[3]}$.

\section{Architecture of software in platform service layer (PaaS)}

Software in platform service layer mainly exists in platform service layer, which can provide users with test, operation, application and development environment. Main representatives of software architecture in platform service layer are Cloud Foundry and Hadoop structures, mainly including node and cloud controller. Client mainly develops software platform of software service layer according to browser interface and command line and provides the corresponding environment of software test. Cloud controller mainly includes dispatcher, user manager, monitor, database manager, platform component manager, application execution engine and cloud interface. Cloud interface is mainly interface for access to cloud computing platform, i.e. interface for user remote control and conversion into API interface. It has user manager, monitor, platform component manager and dispatcher similar to software in basic service layer. Application execution engine mainly initiates nodes in software platform. To protect the progress of application node, all nodes should be isolated. For example, JVM virtual machine needs to be isolated.

In the design, open source software of cloud computing should have data integrity and confidentiality. The main realization way is security management. In the living example of distributed Openstack structure, to guarantee the overall confidentiality of system, data in storage system are encrypted and encrypted data are transmitted with SSL and TLS so as to guarantee the security of data transmission process and then provide transmission data with confidentiality. Data integrity should be actually protected when the authentication of user data is completed. Another open source software platform of cloud computing gaining attention is Eucalyptus, mainly including five parts for registering and guaranteeing system security. Besides data transmission, storage and encryption and the verification of data integrity, it is also necessary to attach importance to cluster controller of the system, which can interact internal and external information and form system attack target. Therefore, it is required to guarantee system security with the following method: do not interrupt the system on the basis of influencing and changing operating efficiency of system; check in time once any abnormal connection occurs when transmission connection process is monitored ${ }^{[4]}$. 
Importance has been attached to security risk of cloud computing. The openness of cloud computing will not reduce system security; instead, it will improve system security. Cloud computing security service includes the following four conditions: first, feasibility. It is guaranteed that computing can provide services for users whenever and wherever possible. Currently, it mainly includes availability service method, virtual technology and redundancy technology. Virtual technology can provide system with resources. Large cloud service systems in the globe have data center, the main function of which is to avoid data corruption influencing the whole data center and conduct data backup. Second, confidentiality. Currently, encryption technology and physical technology under confidentiality way are included. Physical isolation is actually virtual isolation. When public network spreads cloud service, protection wall and VPN are used to guarantee the security of data isolation in data center. Meanwhile, it is required to integrate an enhanced confidentiality technology before the encryption of important data. Third, data integrity. To guarantee that the network will not alter data randomly, it is necessary to guarantee data integrity. Forth, control. In fact, it refers to reasonable allocation of system resources for users. The foundation is virtual technology. Therefore, control technology will be restricted. Control mechanism cannot be provided for system after resource virtualization ${ }^{[5]}$.

\section{Conclusion}

In conclusion, with the constant development of cloud computing, traditional software platform can no longer meet actual demands of users. Future development of open source software of cloud computing becomes especially important. Open source software of cloud computing under ideal conditions has higher security and reliability.

\section{References}

[1] Zhou Xiangbing, Ma Hongjiang, Miao Fang et al. A Cloud Solution for Wisdom Travel Based on Cloud Computing. Journal of Southeast University (Natural Science Edition), 2012,42(z2):261-264.

[2] Zhou Xiangbing, Ma Hongjiang, Miao Fang et al. A Cloud Solution for Wisdom Travel Based on Cloud Computing. Memoir of the 19th National Academic Conference for Network and Data Communication, 2012:84-89.

[3] Di Xiaoqiang, Zhang Yuxin, Zhao Jianping et al. Exploration on Construction of Experimental Teaching Platform for Computer Network Attack and Defense. Experimental Technology and Management, 2015,32(4):147-151.

[4] Che Lu, Zhang Huanyuan, Xia Yadong et al. Design and Realization of Cloud Monitoring Platform Based on Open Source Software. Computing Technology and Automation, 2015(1):136-140.

[5] Chen Delu, Wu Hong. Study on Application of Cloud Computing in Taxation Service Platform. Computer Knowledge and Technology, 2010,06(21):5815-5817. 